\title{
Ultrafast momentum imaging of pseudospin-flip excitations in graphene
}

\author{
S. Aeschlimann, ${ }^{1, *}$ R. Krause, ${ }^{1}$ M. Chávez-Cervantes, ${ }^{1}$ H. Bromberger, ${ }^{1}$ R. Jago, ${ }^{2}$ E. Malić, ${ }^{2}$ A. Al-Temimy, ${ }^{3}$ C. Coletti, ${ }^{3}$ \\ A. Cavalleri, ${ }^{1,4}$ and I. Gierz ${ }^{1, \dagger}$ \\ ${ }^{1}$ Max Planck Institute for the Structure and Dynamics of Matter, Center for Free \\ Electron Laser Science, 22761 Hamburg, Germany \\ ${ }^{2}$ Department of Physics, Chalmers University of Technology, 41258 Gothenburg, Sweden \\ ${ }^{3}$ Center for Nanotechnology @ NEST, Istituto Italiano di Tecnologia, 56127 Pisa, Italy \\ ${ }^{4}$ Department of Physics, Clarendon Laboratory, University of Oxford, Oxford, OX1 3PU, United Kingdom
}

(Received 19 January 2017; revised manuscript received 26 April 2017; published 10 July 2017)

\begin{abstract}
The pseudospin of Dirac electrons in graphene manifests itself in a peculiar momentum anisotropy for photoexcited electron-hole pairs. These interband excitations are in fact forbidden along the direction of the light polarization and are maximum perpendicular to it. Here, we use time- and angle-resolved photoemission spectroscopy to investigate the resulting unconventional hot carrier dynamics, sampling carrier distributions as a function of energy, and in-plane momentum. We first show that the rapidly-established quasithermal electron distribution initially exhibits an azimuth-dependent temperature, consistent with relaxation through collinear electron-electron scattering. Azimuthal thermalization is found to occur only at longer time delays, at a rate that depends on the substrate and the static doping level. Further, we observe pronounced differences in the electron and hole dynamics in $n$-doped samples. By simulating the Coulomb- and phonon-mediated carrier dynamics we are able to disentangle the influence of excitation fluence, screening, and doping, and develop a microscopic picture of the carrier dynamics in photoexcited graphene. Our results clarify new aspects of hot carrier dynamics that are unique to Dirac materials, with relevance for photocontrol experiments and optoelectronic device applications.
\end{abstract}

DOI: 10.1103/PhysRevB.96.020301

The existence of anisotropic photocarrier distributions in graphene was predicted [1,2] and observed in optical pump-probe experiments [3-6], which showed a pronounced difference in the time-dependent optical response for different probe polarizations. The decay of the anisotropy extracted in this manner was attributed to optical phonon emission $[2-5,7,8]$. However, a complete picture for these nonequilibrium phenomena can only be obtained by tracking both carrier energy and momentum in the time domain.

Here we use time- and angle-resolved photoemission spectroscopy (tr-ARPES) at extreme ultraviolet (XUV) wavelengths to track the temporal evolution of the photoexcited carrier distribution as a function of energy and momentum. We establish a hierarchy of events that redistribute carriers on the Dirac cone, including the formation of a quasithermal state with an azimuth-dependent anisotropic electron temperature, which indicates that primary thermalization occurs through collinear electron-electron scattering. Azimuthal relaxation through phonon emission and noncollinear electron-electron scattering plays a role only at later time delays and is found to be strongly influenced by the substrate and the type of static doping of the graphene layer. Furthermore, the finite doping in our samples breaks the electron-hole symmetry and results in different dynamics for electrons and holes. Microscopic simulations of the anisotropic carrier dynamics indicate that the observed dynamics are due to a subtle interplay between doping that affects the scattering phase space and substrate screening which reduces the influence of electron-electron scattering.

\footnotetext{
*sven.aeschlimann@mpsd.mpg.de

†isabella.gierz@mpsd.mpg.de
}

Two different kinds of graphene samples were used for the present investigation. $N$-doped monolayer samples with an equilibrium chemical potential of $\mu_{e}=+0.4 \mathrm{eV}$ and an effective screening constant of $\epsilon=22$ [9] were obtained by thermal decomposition of the silicon face of $\mathrm{SiC}[10,11]$. $P$ doped samples with the chemical potential at $\mu_{e}=-0.2 \mathrm{eV}$ and an effective screening constant of $\epsilon=4.4$ [9] were instead obtained by decoupling the first inactive carbon monolayer formed by thermal decomposition of the same $\mathrm{SiC}$ face by hydrogen intercalation $[11,12]$. After growth, these samples were exposed to air, characterized by Raman spectroscopy, reinserted into ultrahigh vacuum, and cleaned via annealing at $800^{\circ} \mathrm{C}$.

The tr-XUV-ARPES experiments were performed at the MPISD in Hamburg. A Titanium:Sapphire amplifier operating at $1 \mathrm{kHz}$ repetition rate was used to generate synchronized $800 \mathrm{~nm}$ optical pump and XUV probe pulses. The latter were obtained by high harmonic generation in an Argon gas jet. The 17th harmonic at $\hbar \omega_{\text {probe }}=26.3 \mathrm{eV}$ was selected with a time-preserving grating monochromator [13] and used to measure photoelectron distributions from the sample. The probe polarization was fixed along the $x$ axis [Fig. 1(a)]. The polarization of the pump pulses was switched between $x$ and $y$ by rotating a half-wave plate. Both pump and probe impinged onto the sample at normal incidence. The experimental data shown in this work was obtained with pump fluences ranging from 1.3 to $2.8 \mathrm{~mJ} / \mathrm{cm}^{2}$. The energy and temporal resolution of the tr-ARPES experiment were $350 \mathrm{meV}$ and $145 \mathrm{fs}$, respectively.

For the experiments reported here, we used a hemispherical analyzer with the entrance slit parallel to the $x$ axis, to measure the photocurrent as a function of energy and in-plane momentum $k_{x}$ [Fig. 1(a)]. In order to record the complete 
(a)
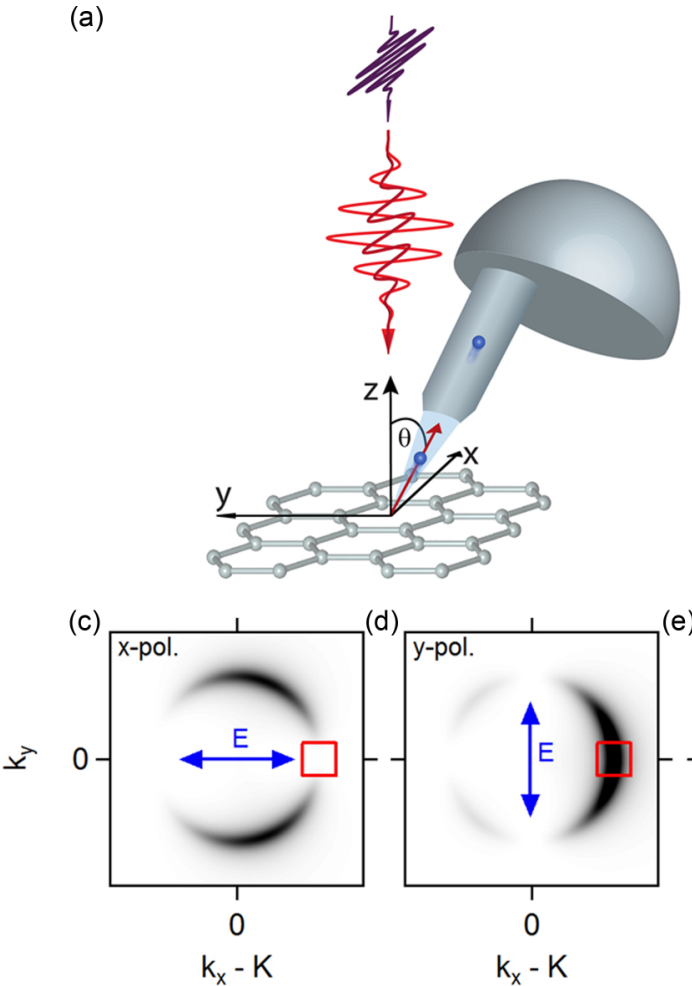

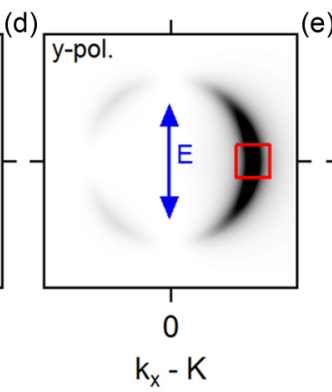

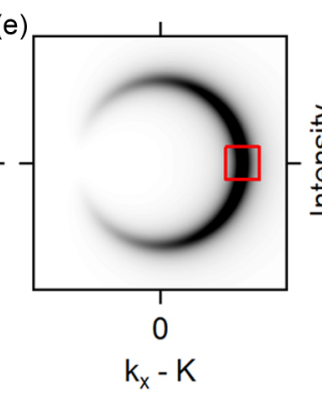

(b)
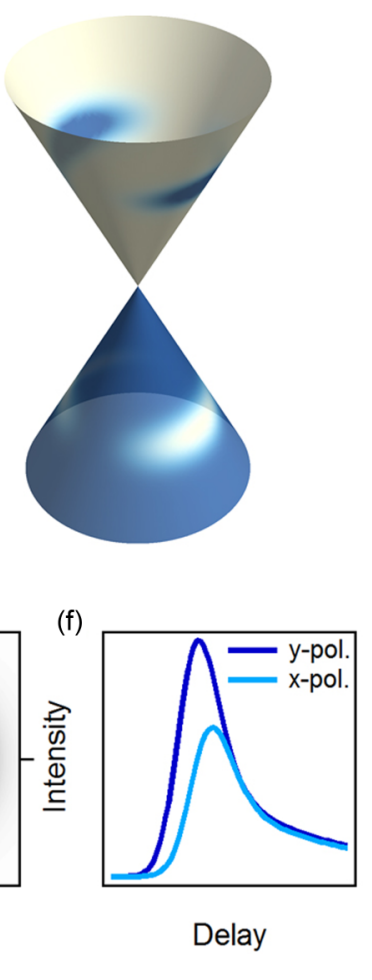

FIG. 1. (a) Sketch of the experimental setup. The sample is excited with $x$ - or $y$-polarized pump pulses (red). Photoelectrons are ejected with $x$-polarized XUV probe pulses (violet) and pass through a hemispherical analyzer. (b) Expected anisotropic charge carrier distribution after photoexcitation of monolayer graphene. Occupied and empty states are shown in blue and white, respectively. (c)-(e) Expected photoemission spectra at constant energy $E=E_{D}+\hbar \omega_{\text {pump }} / 2$ as a function of $k_{x}$ and $k_{y}$ in the first instant after photoexcitation with $x$ - (c) and $y$-polarized light (d) and the expected spectrum of an isotropic distribution (e). (f) Sketch of the expected temporal evolution of the number of carriers inside the red box shown in (c), (d), and (e).

Dirac cone (photocurrent as a function of $k_{x}, k_{y}$, and energy) we rotated the sample around the $x$ axis.

Pump pulses at $\hbar \omega_{\text {pump }}=1.5 \mathrm{eV}$ generated electron-hole pairs at $E_{D} \pm \hbar \omega_{\text {pump }} / 2$, where $E_{D}$ is the energy of the Dirac point where conduction and valence band meet [Fig. 1(b)]. This process mapped valence band states onto conduction band states of opposite pseudospin. Hence, optical excitation involved pseudospin flips which resulted in an angledependent transition probability $\left|M_{\text {pump }}\right|^{2} \propto \sin ^{2}\left(\phi_{k}-\phi_{A}^{\text {pump }}\right)$ $[1,2]$, where $\phi_{k}$ and $\phi_{A}^{\text {pump }}$ are the angles between the $k$ vector of the electron or the pump polarization and the $x$ axis, respectively. As immediately evident from the expression above, the transition probability was then zero along the direction of the electric field $\left(\phi_{k}=\phi_{A}^{\text {pump }}\right)$ and maximum perpendicular to it.

Note also that the photocurrent is subject to momentumdependent matrix element effects. The photoemission cross section in graphene is proportional to $\left|M_{\text {probe }}\right|^{2} \propto 1 / 2(1 \pm$ $\left.\cos \left(\phi_{k}-2 \phi_{A}^{\text {probe }}\right)\right)$ [14-16] with the upper (lower) sign for the conduction (valence) band and $\phi_{A}^{\text {probe }}=0$ in the present experiment, which turns part of the Dirac cone invisible. The photoelectron distribution can then be obtained by multiplying the actual carrier distribution with $\left|M_{\text {probe }}\right|^{2}$.

Figures 1(c)-1(e) illustrate the expected photoelectron spectrum at $E_{D}+\hbar \omega_{\text {pump }} / 2$ as a function of $k_{x}$ and $k_{y}$ for excitation with $x$ - and $y$-polarized light, and, for comparison, for a homogeneous carrier distribution. Figure 1(f) shows the expected evolution in time of the photocurrent inside the red box in Figs. 1(c)-1(e) [2-8]. For pump pulses polarized along the $x$ axis, the carriers are expected to fill these states only after scattering around the cone. Hence, we expect to measure a delayed rise and a lower peak signal for excitation with $x$-polarized light compared to excitation with $y$-polarized light. We also expect the two curves to overlap once the distribution becomes isotropic, before further cooling by optical and acoustic phonon emission occurs at longer time delays [17-25].

In a first set of experiments we measured the photocurrent as a function of energy and $k_{x}$, and compared the effect of $x$ and $y$-polarized excitation in $p$ - and $n$-doped samples (upper and lower panel of Fig. 2, respectively). Figures 2(a) and 2(d) show ARPES snapshots at a negative pump-probe delay and pump-induced changes of the photocurrent at the pump-probe delay at which the signal was maximum. In order to compare the number of excited carriers for $x$ - and $y$-polarized pump pulses we integrated the photocurrent over the area indicated in Figs. 2(a) and 2(d) (white boxes). The time-dependent photocurrent is shown in Figs. 2(b) and 2(e). These data were fitted with an error function and a double exponential decay. We also show the temporal cross correlation between pump and probe pulses (gray-shaded area), as obtained from the temporal derivative of the error function, with a full width at half maximum of $145 \mathrm{fs}$. For $p$-doped samples, the pump-probe signal for $x$ - and $y$-polarized pump pulses was found to be 

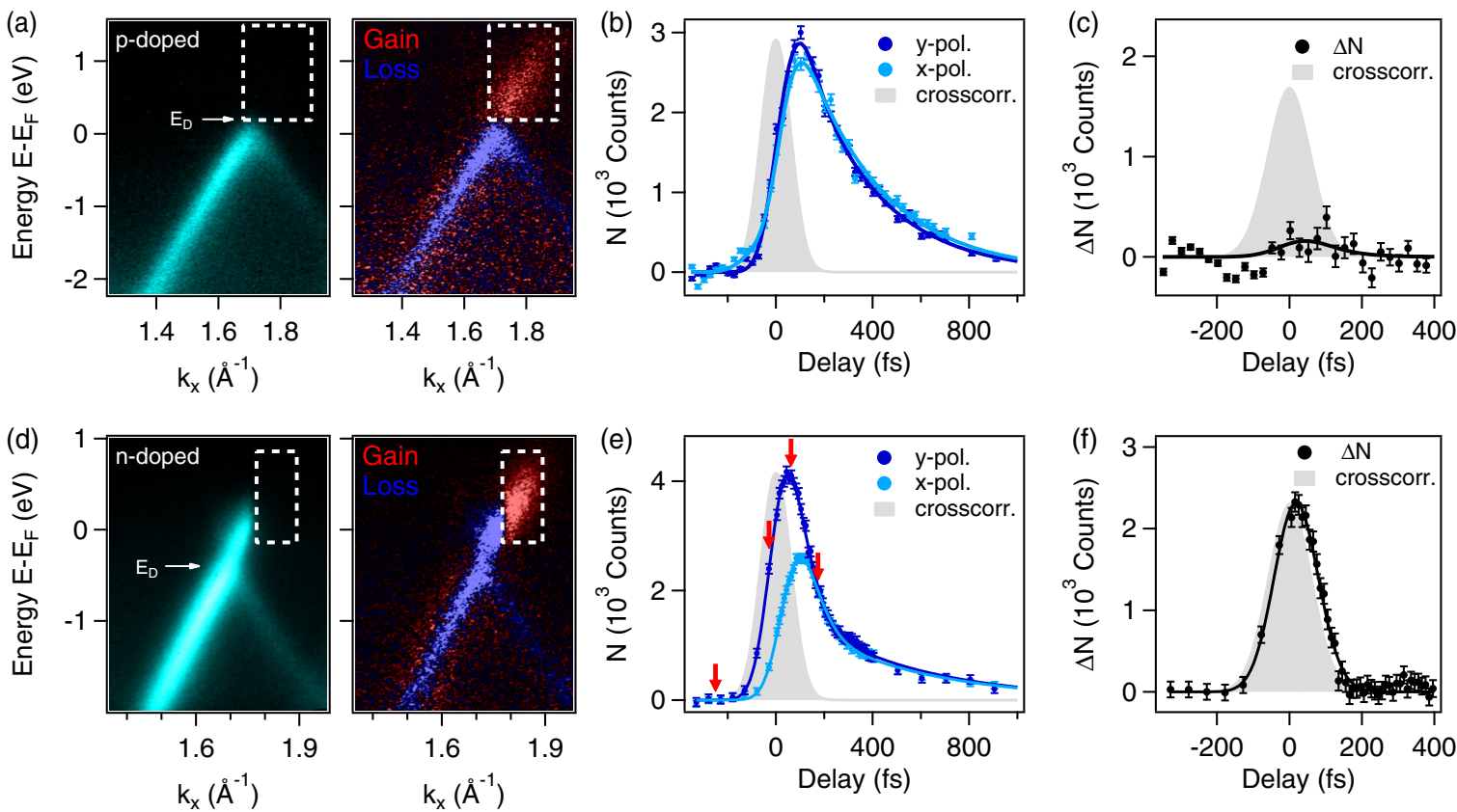

FIG. 2. Photoemission data for $p$-doped (upper panel, excitation fluence of $1.5 \mathrm{~mJ} / \mathrm{cm}^{2}$ ) and $n$-doped graphene (lower panel, excitation fluence of $2.8 \mathrm{~mJ} / \mathrm{cm}^{2}$ ): (a), (d) ARPES spectra for negative time delays and pump-induced changes of the photocurrent for $y$-polarized pump pulses at the peak of the pump-probe signal. (b), (e) photocurrent integrated over the area of the white boxes in (a) and (d) versus pump-probe delay for $x$ - (light blue) and $y$-polarized pump pulses (dark blue). The respective difference in intensity is shown in (c) and (f). The light gray area represents the temporal cross correlation of pump and probe pulses. Tr-ARPES data for the $n$-doped sample for an excitation fluence of $1.3 \mathrm{~mJ} / \mathrm{cm}^{2}$ is shown in Ref. [26].

the same within the error bars. On the contrary, we found a pronounced difference between the two pump polarizations for the $n$-doped sample, indicating the presence of a long-lived anisotropic carrier distribution. In Figs. 2(c) and 2(f) we plot the time-dependent anisotropy [difference between the dark and light blue curves in Figs. 2(b) and 2(e)], which was found to relax at a rate limited by the time resolution of the experiment.

Time-dependent carrier distributions for all $k_{x}$ and $k_{y}$ values were measured for $n$-doped samples and $x$-polarized
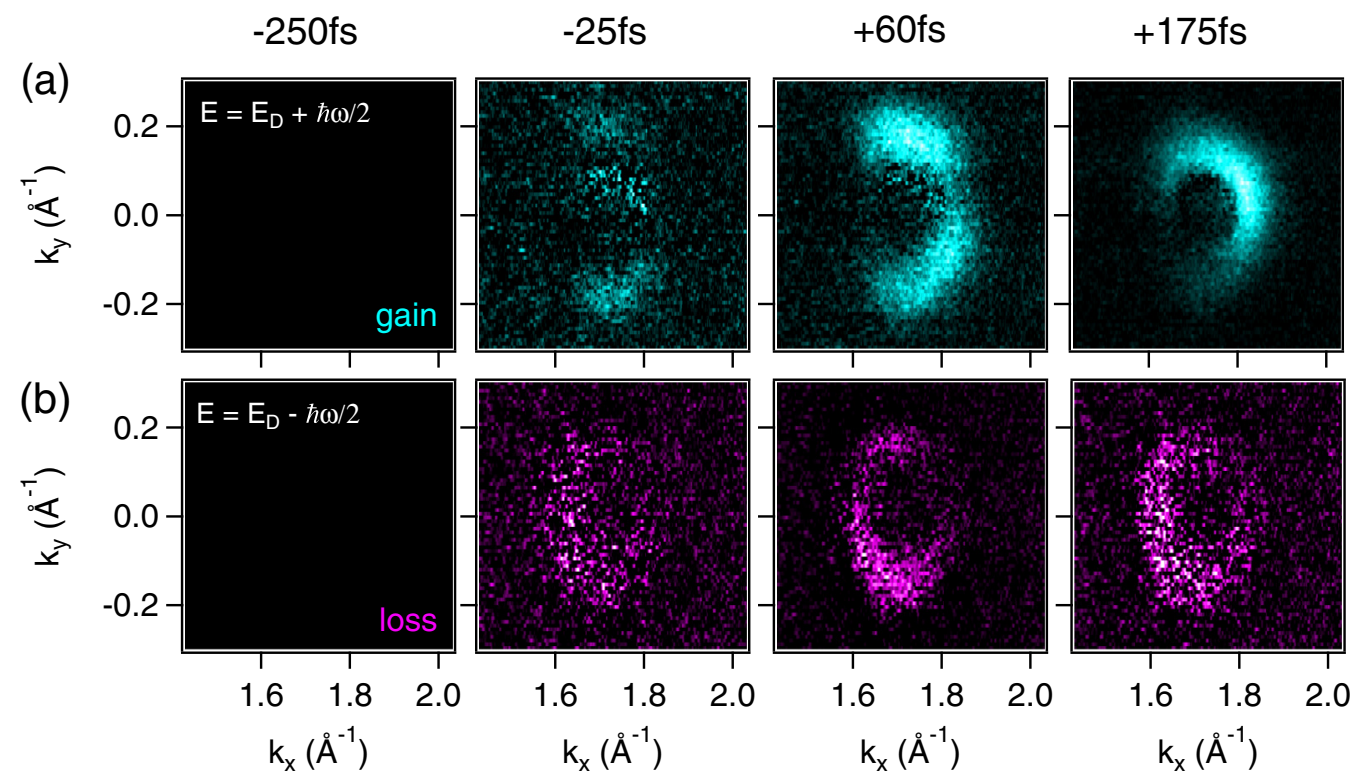

FIG. 3. Photoemission spectra at constant energy $E=E_{D}+\hbar \omega_{\text {pump }} / 2$ [panel (a)] and $E=E_{D}-\hbar \omega_{\text {pump }} / 2$ [panel (b)] as a function of $k_{x}$ and $k_{y}$ for an excitation fluence of $2.8 \mathrm{~mJ} / \mathrm{cm}^{2}$ at four different time delays as indicated by red arrows in Fig. 2(e). Note that the sickle-shaped image at $\mathrm{t}=175 \mathrm{fs}$ is slightly rotated away from the $k_{x}$ axis due to a small azimuthal misalignment of the sample and that the photoemission cross section for the valence band leads to zero intensity on the opposite side of the Dirac cone compared to the conduction band. 

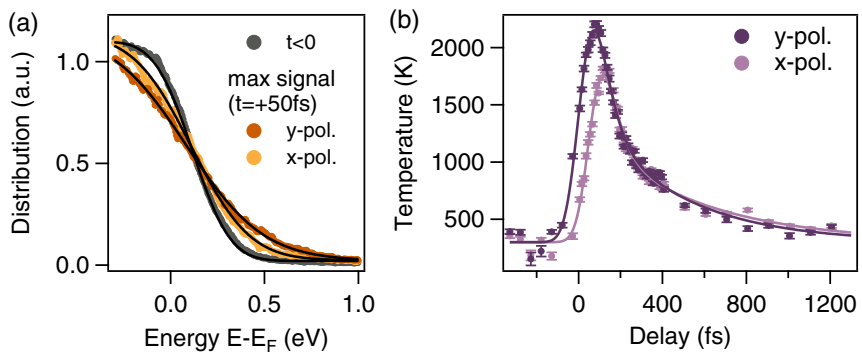

FIG. 4. (a) Electron distribution functions along the $k_{x}$ direction for $n$-doped graphene. Gray curves show the distribution at negative pump-probe delay; light and dark orange curves show the respective distributions at $\mathrm{t}=50 \mathrm{fs}$ for $x$ - and $y$-polarized pump pulses. Black curves are Fermi-Dirac fits. (b) Temporal evolution of the electron temperature obtained from the fits in (a).

pump pulses. Constant-energy cuts integrated over an interval of $\pm 50 \mathrm{meV}$ around $E_{D}+\hbar \omega_{\text {pump }} / 2$ are reported for four different delays [Fig. 3(a)], indicated by red arrows in Fig. 2(e). At negative delay ( $\mathrm{t}=-250 \mathrm{fs})$ no excited carriers are detected. For a time delay of $t=-25$ fs, that is half way through the rising edge, the anisotropic carrier distribution is already observable, reaching its maximum at $t=+60$ fs. At $\mathrm{t}=+175 \mathrm{fs}$ the carrier distribution becomes isotropic, with an angular dependence caused by the photoemission matrix element alone. The measured spectra nicely agree with the expectations shown in Figs. 1(c)-1(e). For comparison, we also show the photoexcited hole distribution at $E_{D}-\hbar \omega_{\text {pump }} / 2$ in Fig. 3(b). Note that the photoemission cross section for the valence band is flipped with respect to the one of the conduction band. Sketches of the expected measured hole distribution can be obtained by mirroring Figs. 1(c)-1(e) on the $k_{y}$ axis. The measured hole distribution [Fig. 3(b)] shows a much smaller anisotropy than the measured electron distribution [Fig. 3(a)]. A more detailed comparison between electron and hole dynamics is given in the Supplemental Material [26].

By integrating the two-dimensional ARPES spectra in Fig. 2(d) along $k_{x}$ for $x$ - and $y$-polarized pump pulses, we obtained transient electron distribution functions [27,28] at the minima and maxima of $\left|M_{\text {pump }}\right|^{2}$, respectively, along the direction where the photoemission cross section is maximum. The gray data points in Fig. 4(a) show the distribution at negative delay. Light and dark orange data points show the distributions for $x$ - and $y$-polarized pump pulses at $\mathrm{t}=$ +50 fs where the pump-probe signal reaches its maximum for excitation with $y$-polarized light. The black lines are Fermi-Dirac fits convolved with a Gaussian with a full width at half maximum of $350 \mathrm{meV}$ to account for the finite energy resolution. The temporal evolution of the resulting electron temperature is shown in Fig. 4(b). At early times, the electron temperature along $k_{x}$ is found to be smaller for $x$-polarized pump pulses than for $y$-polarized pump pulses.

We first note that the electron distribution can be described with a Fermi-Dirac distribution at all pump-probe delays [Fig. 4(a)], indicating that electron-electron scattering thermalizes the photoexcited carriers on a time scale short compared to our temporal resolution. The observed
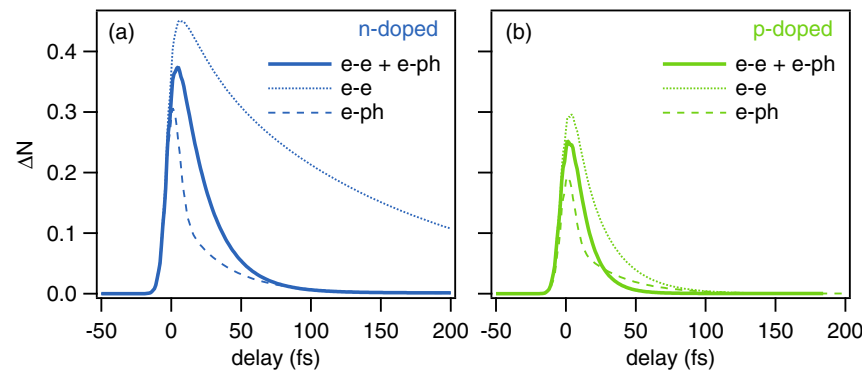

FIG. 5. Simulated dynamics of the anisotropy in the $n$-doped (a) and the $p$-doped sample (b). Solid lines represent the full dynamics; dotted and dashed lines represent the dynamics for electron-electron and electron-phonon scattering only, respectively.

pump-polarization dependence of the electron temperature [Fig. 4(b)] shows that this transient quasithermal state has an azimuth-dependent temperature and provides direct evidence that electron-electron scattering is strongly confined to lines pointing radially away from the Dirac point as predicted in Refs. [2,7].

Relaxation around the cone, which re-establishes an isotropic carrier distribution, can in principle occur through electron-phonon scattering or noncollinear electron-electron scattering. While the decay of the anisotropy is believed to be dominated by phonon emission in the low fluence regime $[2,3,7]$, we expect noncollinear electron-electron scattering to be of similar importance for the high excitation fluences applied in this work. In order to develop a microscopic understanding of the scattering channels that are responsible for the decay of the anisotropy in the present study, we simulate the influence of pump fluence, substrate screening, and doping on the anisotropic carrier dynamics in graphene. Details are given in the Supplemental Material [26]. In Fig. 5 we present the simulated dynamics of the anisotropy for the two different graphene samples for a pump fluence of $1.5 \mathrm{~mJ} / \mathrm{cm}^{2}$. In agreement with the experiment we find a larger and longer-lived anisotropy for the $n$-doped sample [Fig. 5(a)] compared to the $p$-doped sample [Fig. 5(b)]. The reason for the enhanced lifetime of the anisotropy in the $n$-doped sample can be traced back to the large value of the chemical potential that reduces the scattering phase space for both electron-electron [dotted lines in Figs. 5(a) and 5(b)] and electron-phonon scattering [dashed lines in Figs. 5(a) and 5(b)] as well as the strong effective screening of the Coulomb interaction due to the large dielectric constant of the substrate. As the measured lifetime of the anisotropy in the present work is resolution limited, the difference in lifetime shows up as a difference in amplitude of the measured anisotropy. Our microscopic simulations are also able to reproduce the difference between electron and hole dynamics (see Supplemental Material [26]). This can be explained by the finite positive value of the chemical potential that breaks the electron-hole symmetry and increases (decreases) the scattering phase space for holes (electrons).

In summary, we have used time- and angle-resolved photoemission spectroscopy to visualize anisotropic photocarrier distributions in $p$ - and $n$-doped monolayer graphene. We found that collinear electron-electron scattering rapidly 
thermalizes the carriers along lines pointing radially away from the Dirac point, leading to a quasithermal state with an azimuth-dependent electron temperature. We also observed that the magnitude and the decay of the measured anisotropy are influenced by the underlying substrate and the doping level of the graphene layer and are different for electrons and holes. Using microscopic simulations of the anisotropic carrier dynamics we are able to explain the experimental observations by a subtle interplay of doping that modifies the scattering phase space and screening that reduces the efficiency of electron-electron scattering. Our results visualize photocarrier dynamics that are unique to Dirac materials, in which the pseudospin is responsible for peculiar anisotropic photocarrier distributions. We also note that the ability to tune hot carrier dynamics via doping or screening might potentially be exploited in graphene-based thermoelectric devices [42-46] or other opto-electronic applications of this class of solids.

This work received financial support from the German Research Foundation through the Priority Program SPP 1459 and the Collaborative Research Center SFB 925 as well as the European Union's Horizon 2020 Research and Innovation Programme under Grant Agreement No. 696656-GrapheneCore1.
[1] M. Trushin and J. Schliemann, Europhys. Lett. 96, 37006 (2011).

[2] E. Malić, T. Winzer, E. Bobkin, and A. Knorr, Phys. Rev. B 84, 205406 (2011).

[3] M. Mittendorff, T. Winzer, E. Malić, A. Knorr, C. Berger, W. A. de Heer, H. Schneider, M. Helm, and S. Winnerl, Nano Lett. 14, 1504 (2014).

[4] X.-Q. Yan, J. Yao, Z.-B. Liu, X. Zhao, X.-D. Chen, C. Gao, W. Xin, Y. Chen, and J.-G. Tian, Phys. Rev. B 90, 134308 (2014).

[5] M. Trushin, A. Grupp, G. Soavi, A. Budweg, D. De Fazio, U. Sassi, A. Lombardo, A. C. Ferrari, W. Belzig, A. Leitenstorfer, and D. Brida, Phys. Rev. B 92, 165429 (2015).

[6] J. C. König-Otto, M. Mittendorff, T. Winzer, F. Kadi, E. Malić, A. Knorr, C. Berger, W. A. de Heer, A. Pashkin, H. Schneider, M. Helm, and S. Winnerl, Phys. Rev. Lett. 117, 087401 (2016).

[7] E. Malić, T. Winzer, and A. Knorr, Appl. Phys. Lett. 101, 213110 (2012).

[8] A. Satou, V. Ryzhii, and T. Otsuji, J. Phys. Conf. Ser. 584, 012018 (2015).

[9] A. L. Walter, A. Bostwick, K.-J. Jeon, F. Speck, M. Ostler, T. Seyller, L. Moreschini, Y. J. Chang, M. Polini, R. Asgari, A. H. MacDonald, K. Horn, and E. Rotenberg, Phys. Rev. B 84, 085410 (2011).

[10] A. J. Van Bommel, J. E. Crombeen, and A. Van Tooren, Surf. Sci. 48, 463 (1975).

[11] U. Starke, S. Forti, K. V. Emtsev, and C. Coletti, MRS Bull. 37, 1177 (2012).

[12] C. Riedl, C. Coletti, T. Iwasaki, A. A. Zakharov, and U. Starke, Phys. Rev. Lett. 103, 246804 (2009).

[13] F. Frassetto, C. Cacho, C. A. Froud, I. C. E. Turcu, P. Villoresi, W. A. Bryan, E. Springate, and L. Poletto, Opt. Express 19, 19169 (2011).

[14] E. L. Shirley, L. J. Terminello, A. Santoni, and F. J. Himpsel, Phys. Rev. B 51, 13614 (1995).

[15] H. Daimon, S. Imada, H. Nishimoto, and S. Suga, J. Electron Spectrosc. Relat. Phenom. 76, 487 (1995).

[16] A. Bostwick, T. Ohta, J. L. McChesney, K. V. Emtsev, T. Seyller, K. Horn, and E. Rotenberg, New J. Phys. 9, 385 (2007).

[17] J. C. Johannsen, S. Ulstrup, F. Cilento, A. Crepaldi, M. Zacchigna, C. Cacho, I. C. E. Turcu, E. Springate, F. Fromm, C. Raidel, T. Seyller, F. Parmigiani, M. Grioni, and P. Hofmann, Phys. Rev. Lett. 111, 027403 (2013).

[18] M. Breusing, S. Kuehn, T. Winzer, E. Malić, F. Milde, N. Severin, J. P. Rabe, C. Ropers, A. Knorr, and T. Elsaesser, Phys. Rev. B 83, 153410 (2011).
[19] T. Kampfrath, L. Perfetti, F. Schapper, C. Frischkorn, and M. Wolf, Phys. Rev. Lett. 95, 187403 (2005).

[20] H. Yan, D. Song, K. F. Mak, I. Chatzakis, J. Maultzsch, and T. F. Heinz, Phys. Rev. B 80, 121403(R) (2009).

[21] K. Kang, D. Abdula, D. G. Cahill, and M. Shim, Phys. Rev. B 81, 165405 (2010)

[22] T. Winzer, A. Knorr, and E. Malić, Nano Lett. 10, 4839 (2010).

[23] J. C. W. Song, M. Y. Reizer, and L. S. Levitov, Phys. Rev. Lett. 109, 106602 (2012).

[24] M. W. Graham, S.-F. Shi, D. C. Ralph, J. Park, and P. L. McEuen, Nat. Phys. 9, 103 (2013).

[25] A. C. Betz, S. H. Jhang, E. Pallecchi, R. Ferreira, G. Fève, J.-M. Berroir, and B. Plaçais, Nat. Phys. 9, 109 (2013).

[26] See Supplemental Material at http://link.aps.org/supplemental/ 10.1103/PhysRevB.96.020301 for further experimental data and details about the microscopic simulations, including Refs. [29-41].

[27] I. Gierz, J. C. Petersen, M. Mitrano, C. Cacho, I. C. E. Turcu, E. Springate, A. Stöhr, A. Köhler, U. Starke, and A. Cavalleri, Nat. Mater. 12, 1119 (2013).

[28] S. Ulstrup, J. C. Johannsen, M. Grioni, and P. Hofmann, Rev. Sci. Instrum. 85, 013907 (2014).

[29] E. Malić and A. Knorr, Graphene and Carbon Nanotubes: Ultrafast Optics and Relaxation Dynamics, 1st ed. (Wiley-VCH, Hoboken, 2013).

[30] E. Malić, T. Winzer, F. Wendler, and A. Knorr, Microscopic view on ultrafast carrier dynamics in graphene, in Optical Properties of Graphene, edited by R. Binder (World Scientific, Singapore, 2016).

[31] M. Lindberg and S. W. Koch, Phys. Rev. B 38, 3342 (1988).

[32] H. Haug and S. W. Koch, Quantum Theory of the Optical and Electronic Properties of Semiconductors (World Scientific, Singapore, 2004).

[33] F. Rossi and T. Kuhn, Rev. Mod. Phys. 74, 895 (2002).

[34] A. Knorr, S. Hughes, T. Stroucken, and S. W. Koch, Chem. Phys. 210, 27 (1996).

[35] M. Kira and S. W. Koch, Prog. Quantum Electron. 30, 155 (2006).

[36] F. Kadi, T. Winzer, E. Malić, A. Knorr, F. Göttfert, M. Mittendorff, S. Winnerl, and M. Helm, Phys. Rev. Lett. 113, 035502 (2014).

[37] A. Grüneis, R. Saito, G. G. Samsonidze, T. Kimura, M. A. Pimenta, A. Jorio, A. G. Souza Filho, G. Dresselhaus, and M. S. Dresselhaus, Phys. Rev. B 67, 165402 (2003). 
[38] E. Malić, M. Hirtschulz, F. Milde, A. Knorr, and S. Reich, Phys. Rev. B 74, 195431 (2006).

[39] S. Reich, J. Maultzsch, C. Thomsen, and P. Ordejón, Phys. Rev. B 66, 035412 (2002).

[40] F. Kadi, T. Winzer, A. Knorr, and E. Malić, Sci. Rep. 5, 16841 (2015).

[41] E. Malić, C. Weber, M. Richter, V. Atalla, T. Klamroth, P. Saalfrank, S. Reich, and A. Knorr, Phys. Rev. Lett. 106, 097401 (2011).

[42] T. Mueller, F. Xia, and P. Avouris, Nat. Photonics 4, 297 (2010).
[43] F. Bonaccorso, Z. Sun, T. Hasan, and A. C. Ferrari, Nat. Photonics 4, 611 (2010).

[44] N. M. Gabor, J. C. W. Song, Q. Ma, N. L. Nair, T. Taychatanapat, K. Watanabe, T. Taniguchi, L. S. Levitov, and P. Jarillo-Herrero, Science 334, 648 (2011).

[45] D. Sun, G. Aivazian, A. M. Jones, J. S. Ross, W. Yao, D. Cobden, and X. Xu, Nat. Nanotechnol. 7, 114 (2012).

[46] T. J. Echtermeyer, P. S. Nene, M. Trushin, R. V. Gorbachev, A. L. Eiden, S. Milana, Z. Sun, J. Schliemann, E. Lidorikis, K. S. Novoselov, and A. C. Ferrari, Nano Lett. 14, 3733 (2014). 